\title{
Comparison of Predicted Postoperative Lung Function in Pneumonectomy Using Computed Tomography and Lung Perfusion Scans
}

\author{
Hee Joon Kang, M.D., Seok Soo Lee, M.D. \\ Department of Thoracic and Cardiovascular Surgery, Yeungnam University College of Medicine, Daegu, Korea
}

\section{ARTICLE INFO}

Received July 22, 2021

Revised October 7, 2021

Accepted October 8, 2021

Corresponding author

Seok Soo Lee

Tel $82-53-620-4368$

Fax 82-53-626-8660

E-mail andrea0710@naver.com

ORCID

https://orcid.org/0000-0002-4402-0885
Background: Predicting postoperative lung function after pneumonectomy is essential. We retrospectively compared postoperative lung function to predicted postoperative lung function based on computed tomography (CT) volumetry and perfusion scintigraphy in patients who underwent pneumonectomy.

Methods: Predicted postoperative lung function was calculated based on perfusion scintigraphy and CT volumetry. The predicted function was compared to the postoperative lung function in terms of forced vital capacity (FVC) and forced expiratory volume in 1 second $\left(\mathrm{FEV}_{1}\right)$, using 4 parameters: FVC, FVC\%, FEV , and FEV ${ }_{1} \%$.

Results: The correlations between postoperative function and predicted function based on CT volumetry were $r=0.632$ ( $p=0.003$ ) for FVC\% and $r=0.728$ ( $p<0.001)$ for $\mathrm{FEV}_{1} \%$. The correlations between postoperative function and predicted postoperative function based on perfusion scintigraphy were $r=0.654(p=0.002)$ for $F V C \%$ and $r=0.758(p<0.001)$ for FEV $\%$. The preoperative Eastern Cooperative Oncology Group (ECOG) scores were significantly higher in the group in which the gap between postoperative $\mathrm{FEV}_{1}$ and predicted postoperative FEV analyzed by $C T$ was smaller than the gap analyzed by perfusion scintigraphy ( $1.2 \pm 0.62$ vs. $0.4 \pm 0.52, p=0.006)$.

Conclusion: This study affirms that CT volumetry can replace perfusion scintigraphy for preoperative evaluation of patients needing pneumonectomy. In particular, it was found to be a better predictor of postoperative lung function for poor-performance patients (i.e., those with high ECOG scores).

Keywords: Lung volume measurements, Pneumonectomy, Lung surgery, Computed tomography

\section{Introduction}

With recent advances in medical examinations, diagnostic imaging, targeted chemotherapy, and radiotherapy technology, the number of cases of pneumonectomy is decreasing. However, many patients still undergo pneumonectomy for locally advanced lung cancer or for severe inflammatory lung disease. Pneumonectomy has a high incidence of pulmonary complications due to the rapid and meaningful decrease in lung capacity after surgery, which adversely affects cardiovascular hemodynamics and results in high morbidity and mortality [1]. Therefore, the prediction of postoperative lung function is important for under- standing patients' short-term and long-term course. There are several ways to predict postoperative lung function, including calculation of lung function by counting the residual lobes, calculating residual lung function with perfusion scintigraphy, or using the calculated area obtained by imaging [2]. Perfusion scintigraphy is a relatively accurate method and is widely used. However, for patients in poor condition who cannot undergo preoperative perfusion scintigraphy, it is sometimes necessary to predict the postoperative residual lung function using a different method. A more convenient way to accurately predict postoperative lung function is needed. However, there are few studies on the use of computed tomography (CT) volumetry to pre- 
dict lung function after lobectomy [3-6]. In this study, postoperative lung function and predicted postoperative lung function were compared after using perfusion scintigraphy and CT volumetry. The correlation between the 2 methods was analyzed according to several other factors described below.

\section{Methods}

This retrospective study was conducted using data from patients who underwent pneumonectomy for lung cancer or inflammatory lung disease from January 1, 2004 to December 31, 2019, including preoperative lung function tests, perfusion scintigraphy, CT scans, and postoperative lung function tests. Postoperative lung function tests were performed at least 6 months after surgery. All operations were performed through posterolateral thoracotomy. The patient's age, sex, body mass index (BMI), Eastern Cooperative Oncology Group (ECOG) performance status, smoking history, past medical history, respiratory disease, and reason for surgery were identified. The patients had CT scans in the supine position in the deep inspiratory breath-holding state. The images were $3-\mathrm{mm}$ thick and in a $512 \times 512$ matrix. The area of the pleural cavity was calculated from the CT images. To exclude cancerous lesions, fibrotic tissue, atelectasis, and emphysematous areas, the range was set between -600 Hounsfield unit (HU) and -910 HU [6-8]. The area was calculated for each slice of the CT images (Fig. 1). The volume of each slice was computed by multiplying the area by the 3-mm thickness. The total lung volume was obtained by integrating all slice volumes. This study was approved by the Institutional Review Board (IRB) of Yeungnam University Medical Center (IRB approval no., YUMC-2020-05-094-002). The requirement for informed consent from individual patients was omitted since it was to be conducted on existing anonymous data from previous years.

\section{Prediction of postoperative lung function}

This study compared the postoperative lung function (POST) and the predicted postoperative lung function (PPO) using forced vital capacity (FVC) and forced expiratory volume in 1 second $\left(\mathrm{FEV}_{1}\right)$. However, there was significant variability in when patients underwent postoperative lung function testing. In addition, lung function may differ depending on patient age, height, and weight. Therefore, we compared the POST and PPO using FVC\% and $\mathrm{FEV}_{1} \%$ to compensate for age and other patient-specific factors.

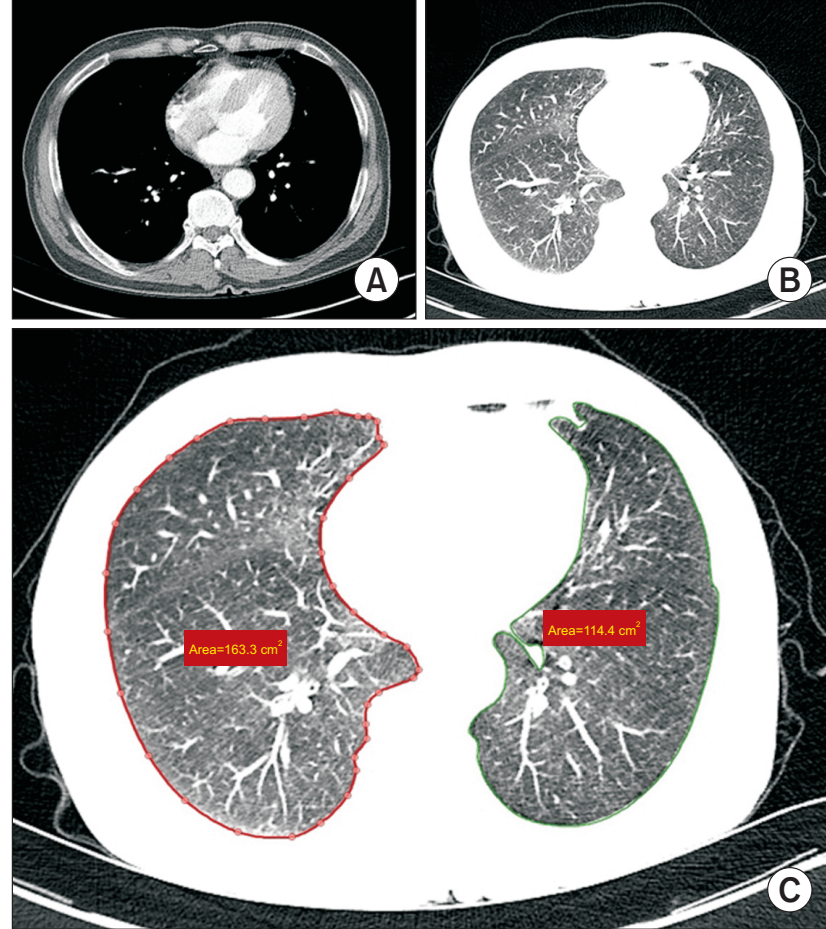

Fig. 1. Process of measuring lung volume from computed tomography $(\mathrm{CT})$ scans. (A) A slice of a CT image. (B) CT image with Hounsfield unit range setting. (C) The left and right lung areas are measured separately.

Other factors that could affect the difference between POST and PPO were also analyzed. Based on preoperative lung function (PRE), PPO was calculated using perfusion scintigraphy and CT volumetry. PPO based on perfusion scintigraphy (PPO-PS) was calculated by the equation $\mathrm{PPO}=\mathrm{PRE} \times$ perfusion ratio of the remaining lungs. $\mathrm{PPO}$ based on CT volumetry (PPO-CT) was calculated by the equation $\mathrm{PPO}=\mathrm{PRE} \times$ residual lung volume/total lung volume [4]. PPO (\%) was calculated by the equation PPO $(\%)=\mathrm{PPO}(\mathrm{l}) \times \mathrm{PRE}(\%) / \mathrm{PRE}(\mathrm{l})$.

\section{Statistical analysis}

PASW SPSS ver. 18.0 (SPSS Inc., Chicago, IL, USA) was used for statistical analysis and a p-value of $\leq 0.05$ was considered to indicate statistical significance.

\section{Results}

\section{Patient characteristics}

This study included 16 men and 4 women, with an average age of 59.7 years. The average BMI was $22.86 \mathrm{~kg} / \mathrm{m}^{2}$. 
There were 13 patients with a history of smoking and 17 patients with a history of respiratory diseases, such as asthma, tuberculosis, and chronic obstructive pulmonary disease. Fourteen patients underwent left pneumonectomy, and 6 patients underwent right pneumonectomy. Fifteen patients underwent surgery for lung cancer and 5 patients for inflammatory lung disease. The average interval from the operation date to the postoperative lung function test was 32.1 months (Table 1). We compared the PRE FVC with the lung volume measured from CT volumetry. The PRE FVC showed a high correlation with the CT-measured lung volume $(\mathrm{r}=0.779, \mathrm{p}<0.001)$ (Fig. 2).

\section{Comparison between postoperative and predicted postoperative lung function}

For FVC, the PPO-PS FVC ranged from 1.24 to $3.20 \mathrm{~L}$, the PPO-CT FVC ranged from 1.26 to $3.12 \mathrm{~L}$, and the

Table 1. Characteristics of patients in the present study

\begin{tabular}{lc}
\hline \multicolumn{1}{c}{ Characteristic } & Value \\
\hline Total no. of patients & 20 \\
Age $(\mathrm{yr})$ & $59.7(43-73)$ \\
Sex (male:female) & $16: 4$ \\
Body mass index $\left(\mathrm{kg} / \mathrm{m}^{2}\right)$ & $22.86(16.9-28.2)$ \\
Resected site (left:right) & $14: 6$ \\
Cause of pneumonectomy & 15 \\
$\quad$ Lung cancer & 5 \\
$\quad$ Infectious disease & $32 \mathrm{mo}(6 \mathrm{mo}-10 \mathrm{yr})$ \\
\hline Time to PFT follow-up
\end{tabular}

Values are presented as number or mean (range).

PFT, pulmonary function test.

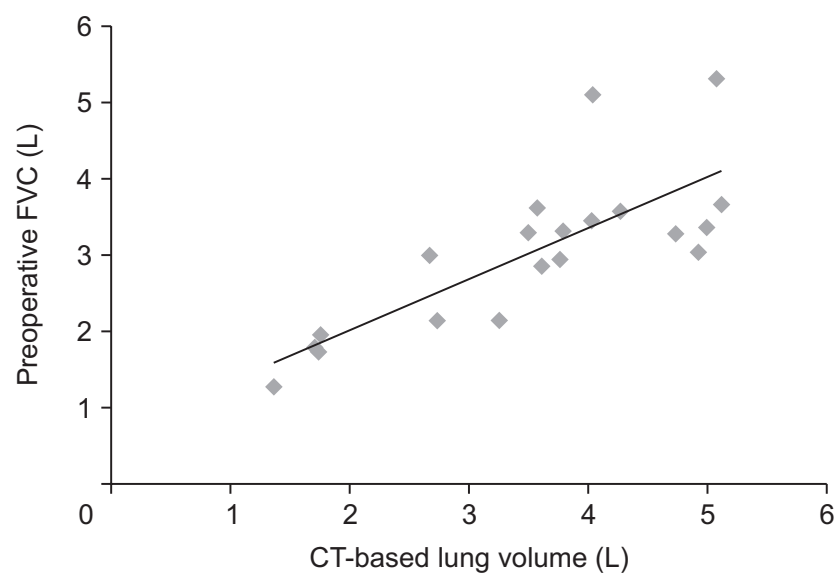

Fig. 2. Comparison between the preoperative forced volume capacity (FVC) and the lung volume calculated with computed tomography $(\mathrm{CT})$ volumetry.
POST FVC ranged from 1.02 to $3.41 \mathrm{~L}$. Both PPO-PS FVC and PPO-CT FVC showed a high correlation with the POST FVC $(r=0.781$ and $r=0.746$, respectively; $\mathrm{p}<0.001)$. For $\mathrm{FEV}_{1}$, the PPO-PS FEV 1 ranged from 0.92 to $2.19 \mathrm{~L}$, the PPO-CT FEV 1 ranged from 0.89 to $1.94 \mathrm{~L}$, and the POST $\mathrm{FEV}_{1}$ ranged from 0.77 to $2.55 \mathrm{~L}$. Both PPO-PS FEV $\mathrm{F}_{1}$ and PPO-CT FEV 1 showed a high correlation with the POST $\mathrm{FEV}_{1}(\mathrm{r}=0.779$ and $\mathrm{r}=0.749$, respectively; $\mathrm{p}<0.001)$ (Table 2). For FVC\%, the PPO-PS FVC\% ranged from $31 \%$ to $77 \%$, the PPO-CT FVC\% ranged from $28 \%$ to $68 \%$, and the POST FVC\% ranged from $29 \%$ to $80 \%$. Both PPO-PS FVC\% and PPO-CT FVC\% showed a high correlation with POST FVC\% ( $r=0.654$ and $r=0.632$, respectively; $\mathrm{p}=0.002$ and 0.003 , respectively) (Fig. 3). For $\mathrm{FEV}_{1} \%$, the PPO-PS $\mathrm{FEV}_{1} \%$ ranged from $30 \%$ to $73 \%$, the PPO-CT $\mathrm{FEV}_{1} \%$ ranged from $27 \%$ to $65 \%$, and the POST $\mathrm{FEV}_{1} \%$ ranged from $22 \%$ to $81 \%$. Both PPO-PS FEV $\%$ and PPO-CT $\mathrm{FEV}_{1} \%$ showed a high correlation with POST $\mathrm{FEV}_{1} \%$ $(r=0.758$ and $r=0.728$, respectively; $\mathrm{p}<0.001)$ (Fig. 4).

Through a comparison of lung function data, the gap between POST and PPO-PS and the gap between POST and PPO-CT were calculated (Table 3 ). On this basis, we divided patients into 2 groups. In the first group, the gap between POST and PPO-PS was smaller than the gap between POST and PPO-CT, and in the second group, the gap between POST and PPO-PS was larger than the gap between POST and PPO-CT. When considering FVC\%, 10 patients had a smaller gap with PPO-PS and the other 10 patients had a smaller gap with PPO-CT. When the first 10 -patient group and the second 10-patient group were compared, the preoperative ECOG scores were significantly different ( 1.20 and 0.40 , respectively; $\mathrm{p}=0.006$ ), and the postoperative ECOG scores were significantly different ( 1.30 and 0.10 , respectively; $\mathrm{p}=0.0003$ ). There were no significant differences in other factors (Table 4). For $\mathrm{FEV}_{1} \%$,

Table 2. Correlations between the predicted postoperative values and measured values in pulmonary function tests

\begin{tabular}{lcrrrr}
\hline \multirow{2}{*}{ Variable } & \multicolumn{2}{c}{ PPO-PS } & & \multicolumn{2}{c}{ PPO-CT } \\
\cline { 2 - 3 } \cline { 5 - 6 } & \multicolumn{1}{c}{$r$} & p-value & & $r$ & p-value \\
\hline FVC & 0.781 & $<0.001^{*}$ & & 0.746 & $<0.001^{*}$ \\
FVC & 0.654 & $0.002^{*}$ & & 0.632 & $0.003^{*}$ \\
FEV $_{1}$ & 0.779 & $<0.001^{*}$ & & 0.749 & $<0.001^{*}$ \\
FEV $_{1}$ & 0.758 & $<0.001^{*}$ & & 0.728 & $<0.001^{*}$ \\
\hline
\end{tabular}

r, Pearson correlation coefficient; PPO-PS, predicted postoperative lung function based on perfusion scintigraphy; FVC, forced vital capacity; $\mathrm{FEV}_{1}$, forced expiratory volume in 1 second; PPO-CT, predicted postoperative lung function based on computed tomography volumetry. ${ }^{*} \mathrm{p}<0.05$ (statistically significant). 

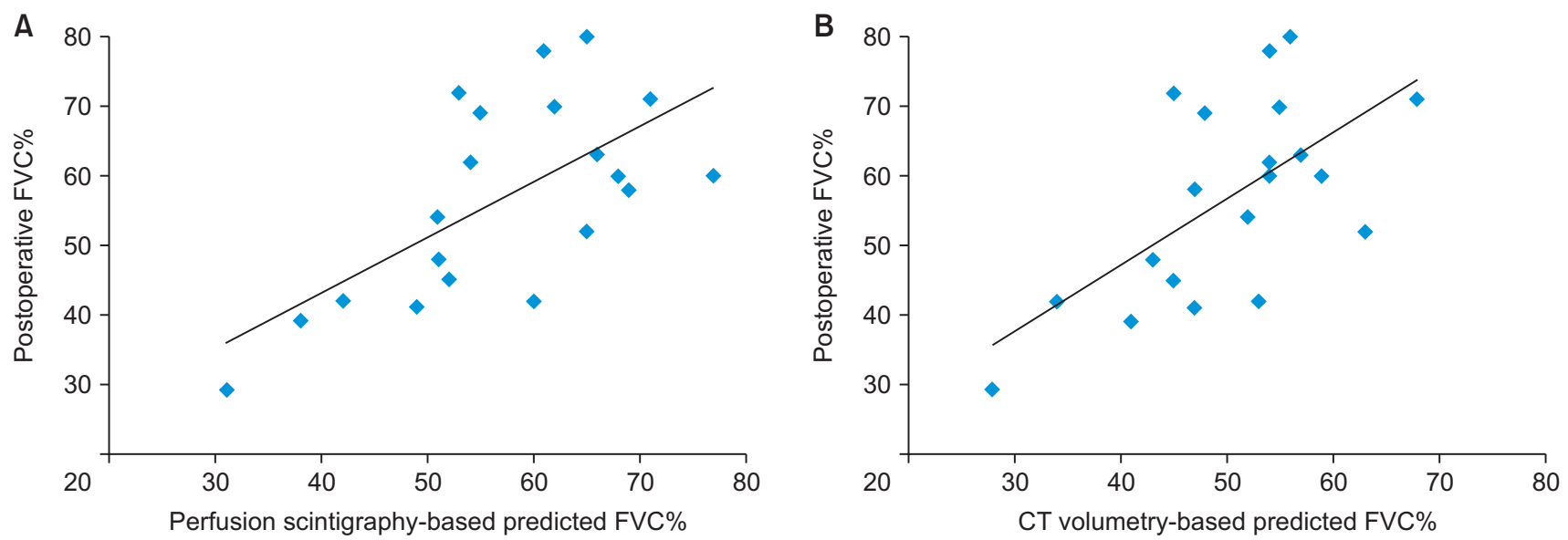

Fig. 3. (A, B) Comparison between postoperative and predicted lung function (FVC\%). FVC, forced volume capacity; CT, computed tomography.
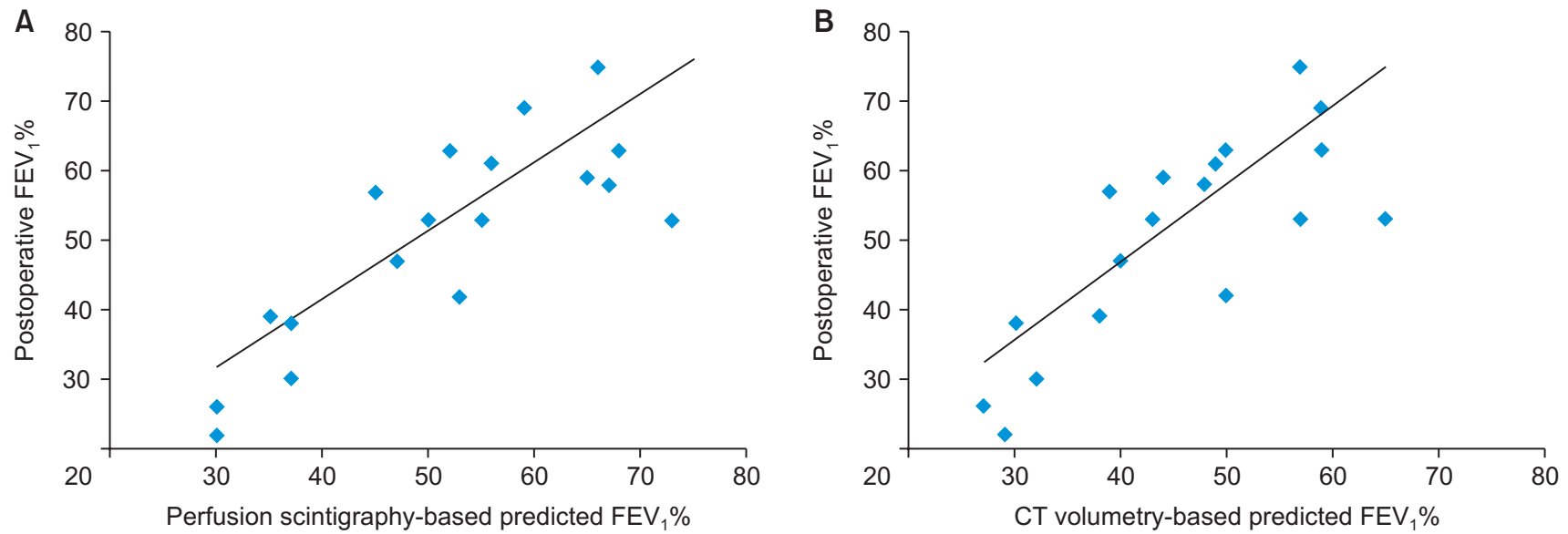

Fig. 4. (A, B) Comparison between postoperative and predicted postoperative lung function ( $\left.\mathrm{FEV}_{1} \%\right)$. FEV ${ }_{1}$, forced expiratory volume in 1 second; CT, computed tomography.

12 patients had a smaller gap with PPO-PS and the other 8 patients had a smaller gap with PPO-CT. When the former and the latter were compared, the preoperative ECOG scores were significantly different (1.25 and 0.50 , respectively; $\mathrm{p}=0.014)$, as were the postoperative ECOG scores ( 1.50 and 0.17 , respectively; $\mathrm{p}=0.0004$ ). There were no significant differences in other factors (Table 5).

\section{Discussion}

When preparing for lung resection surgery, especially pneumonectomy, predicting pulmonary functional reserve is important in decision-making. There are several ways to predict pulmonary functional reserve including segment counting (the Juhl and Frost formula), the split-lung function test (the Kristersson/Olsen formula), and perfusion scintigraphy. For many years, perfusion scintigraphy has been the gold standard for estimating postoperative lung function. Recently, several trials were conducted using CT volumetry [3-6]. As technology has advanced, CT volumetric analysis has provided better estimations of predicted postoperative lung function than the methods previously mentioned [3]. The development of programs that work with CT data has facilitated the construction of 3-dimensional (3D) models, allowing resected lung volume to be calculated from the virtual boundaries of the 3D model [6]. Ueda et al. [6] set the Hounsfield unit range to exclude non-functional parts of the lung such as atelectasis, organized lesions, and emphysematous areas. The results were sufficient to replace perfusion scintigraphy. In this study, the CT volumetric analysis was applied to patients who underwent pneumonectomy and the results obtained were 
Table 3. Lung function test data and calculated gaps between POST and PPO-PS and between POST and PPO-CT

\begin{tabular}{|c|c|c|c|c|c|c|c|c|c|c|c|c|}
\hline \multirow{3}{*}{ No. } & \multicolumn{2}{|c|}{ PRE (\%) } & \multicolumn{2}{|c|}{ POST (\%) } & \multicolumn{4}{|c|}{ PPO-PS } & \multicolumn{4}{|c|}{ PPO-CT } \\
\hline & \multirow{2}{*}{ FVC } & \multirow{2}{*}{$\mathrm{FEV}_{1}$} & \multirow{2}{*}{ FVC } & \multirow{2}{*}{$\mathrm{FEV}_{1}$} & \multicolumn{2}{|c|}{ FVC } & \multicolumn{2}{|c|}{$\mathrm{FEV}_{1}$} & \multicolumn{2}{|c|}{ FVC } & \multicolumn{2}{|c|}{$\mathrm{FEV}_{1}$} \\
\hline & & & & & $\%$ & Gap & $\%$ & Gap & $\%$ & Gap & $\%$ & Gap \\
\hline 1 & 73 & 34 & 52 & 22 & 65 & -13 & 30 & -8 & 63 & -11 & 29 & -7 \\
\hline 2 & 38 & 37 & 29 & 26 & 31 & -2 & 30 & -4 & 28 & 1 & 27 & -1 \\
\hline 3 & 64 & 61 & 72 & 53 & 53 & 19 & 50 & 3 & 45 & 27 & 43 & 10 \\
\hline 4 & 81 & 89 & 62 & 69 & 54 & 8 & 59 & 10 & 54 & 8 & 59 & 10 \\
\hline 5 & 88 & 79 & 70 & 61 & 62 & 8 & 56 & 5 & 55 & 15 & 49 & 12 \\
\hline 6 & 80 & 74 & 48 & 47 & 51 & -3 & 47 & 0 & 43 & 5 & 40 & 7 \\
\hline 7 & 50 & 54 & 41 & 42 & 49 & -8 & 53 & -11 & 47 & -6 & 50 & -8 \\
\hline 8 & 108 & 108 & 63 & 75 & 66 & -3 & 66 & 9 & 57 & 6 & 57 & 18 \\
\hline 9 & 122 & 122 & 60 & 63 & 68 & -8 & 68 & -5 & 59 & 1 & 59 & 4 \\
\hline 10 & 97 & 95 & 78 & 81 & 61 & 17 & 60 & 21 & 54 & 24 & 52 & 29 \\
\hline 11 & 72 & 67 & 58 & 59 & 69 & -11 & 65 & -6 & 47 & 11 & 44 & 15 \\
\hline 12 & 87 & 95 & 54 & 53 & 51 & 3 & 55 & -2 & 52 & 2 & 57 & -4 \\
\hline 13 & 66 & 81 & 42 & 53 & 60 & -18 & 73 & -20 & 53 & -11 & 65 & -12 \\
\hline 14 & 84 & 86 & 69 & 81 & 55 & 14 & 56 & 25 & 48 & 21 & 49 & 32 \\
\hline 15 & 69 & 48 & 80 & 57 & 65 & 15 & 45 & 12 & 56 & 24 & 39 & 18 \\
\hline 16 & 96 & 84 & 60 & 58 & 77 & -17 & 67 & -9 & 54 & 6 & 48 & 10 \\
\hline 17 & 120 & 88 & 71 & 63 & 71 & 0 & 52 & 11 & 68 & 3 & 50 & 13 \\
\hline 18 & 83 & 77 & 39 & 39 & 38 & 1 & 35 & 4 & 41 & -2 & 38 & 1 \\
\hline 19 & 52 & 37 & 45 & 30 & 52 & -7 & 37 & -7 & 45 & 0 & 32 & -2 \\
\hline 20 & 69 & 61 & 42 & 38 & 42 & 0 & 37 & 1 & 34 & 8 & 30 & 8 \\
\hline
\end{tabular}

POST, postoperative lung function; PPO-PS, predicted postoperative lung function based on perfusion scintigraphy; PPO-CT, predicted postoperative lung function based on computed tomography volumetry; PRE, preoperative lung function; FVC, forced volume capacity; FEV ${ }_{1}$, forced expiratory volume in 1 second.

Table 4. Comparison between postoperative FVC\% and predicted FVC\%

\begin{tabular}{|c|c|c|c|}
\hline \multirow{2}{*}{ Characteristic } & \multicolumn{2}{|c|}{ Gap between POST and PPO } & \multirow{2}{*}{ p-value } \\
\hline & $\mathrm{PS}<\mathrm{CT}$ & $\mathrm{PS}>\mathrm{CT}$ & \\
\hline No. of patients & 10 & 10 & \\
\hline Sex (male:female) & $8: 2$ & $8: 2$ & 1.000 \\
\hline Age (yr) & $60.9 \pm 9.3$ & $58.5 \pm 7.6$ & 0.535 \\
\hline Body mass index $\left(\mathrm{kg} / \mathrm{m}^{2}\right)$ & $22.82 \pm 2.73$ & $22.89 \pm 3.18$ & 0.958 \\
\hline Smoking history $(\%)$ & 60 & 70 & 0.660 \\
\hline $\begin{array}{l}\text { Distant history of } \\
\text { tuberculosis (\%) }\end{array}$ & 30 & 40 & 0.660 \\
\hline Hypertension (\%) & 20 & 10 & 0.556 \\
\hline Diabetes mellitus (\%) & 30 & 30 & 1.000 \\
\hline Cancer:infection & $8: 2$ & $7: 3$ & 0.628 \\
\hline Left:right & $7: 3$ & $7: 3$ & 1.000 \\
\hline Preoperative ECOG score & $0.4 \pm 0.52$ & $1.2 \pm 0.63$ & $0.006^{*}$ \\
\hline Postoperative ECOG score & $0.1 \pm 0.32$ & $1.3 \pm 0.95$ & $0.003^{*}$ \\
\hline Preoperative FVC (\%) & $86.2 \pm 17.9$ & $73.7 \pm 24.6$ & 0.210 \\
\hline
\end{tabular}

Values are presented as number or mean \pm standard deviation.

FVC, forced vital capacity; POST, postoperative lung function; PPO, predicted postoperative lung function; PS, perfusion scintigraphy; $\mathrm{CT}$, computed tomography volumetry; ECOG, Eastern Cooperative Oncology Group performance status.

${ }^{*} \mathrm{p}<0.05$ (statistically significant).
Table 5. Comparison between postoperative $\mathrm{FEV}_{1} \%$ and predicted $\mathrm{FEV}_{1} \%$

\begin{tabular}{|c|c|c|c|}
\hline \multirow{2}{*}{ Characteristic } & \multicolumn{2}{|c|}{ Gap between POST and PPO } & \multirow{2}{*}{$p$-value } \\
\hline & $\mathrm{PS}<\mathrm{CT}$ & $\mathrm{PS}>\mathrm{CT}$ & \\
\hline No. of patients & 12 & 8 & \\
\hline Sex (male:female) & $10: 2$ & $6: 2$ & 0.669 \\
\hline Age (yr) & $59.9 \pm 7.7$ & $59.4 \pm 9.8$ & 0.892 \\
\hline Body mass index $\left(\mathrm{kg} / \mathrm{m}^{2}\right)$ & $22.78 \pm 2.58$ & $22.96 \pm 3.48$ & 0.896 \\
\hline Smoking history $(\%)$ & 67 & 63 & 0.858 \\
\hline $\begin{array}{l}\text { Distant history of } \\
\text { tuberculosis (\%) }\end{array}$ & 25 & 50 & 0.274 \\
\hline Hypertension (\%) & 8 & 25 & 0.332 \\
\hline Diabetes mellitus (\%) & 42 & 13 & 0.151 \\
\hline Cancer:infection & $10: 2$ & $5: 3$ & 0.317 \\
\hline Left:right & $10: 2$ & $4: 4$ & 0.156 \\
\hline Preoperative ECOG score & $0.5 \pm 0.52$ & $1.3 \pm 0.710$ & $0.014^{*}$ \\
\hline Postoperative ECOG score & $0.2 \pm 0.39$ & $1.5 \pm 0.926$ & $0.004^{*}$ \\
\hline Preoperative FEV $(\%)$ & $78.8 \pm 17.3$ & $66.4 \pm 31.3$ & 0.329 \\
\hline
\end{tabular}

Values are presented as number or mean \pm standard deviation.

$\mathrm{FEV}_{1}$, forced expiratory volume in 1 second; POST, postoperative lung function; PPO, predicted postoperative lung function; PS, perfusion scintigraphy; CT, computed tomography volumetry; ECOG, Eastern Cooperative Oncology Group performance status.

* $\mathrm{p}<0.05$ (statistically significant). 
more accurate. For FVC\%, PPO-CT had a high correlation with POST ( $\mathrm{r}=0.632, \mathrm{p}=0.003)$. For $\mathrm{FEV}_{1} \%$, PPO-CT had a high correlation with POST $(\mathrm{r}=0.728, \mathrm{p}<0.001)$. Additionally, preoperative and postoperative ECOG scores were higher in patients whose gap between PPO-PS and POST was larger than the gap between PPO-CT and POST.

Our center routinely takes chest CT images while evaluating lung lesions. Eliminating additional scans such as perfusion scintigraphy would help to reduce costs. Patients requiring pneumonectomy are typically in poor condition, with high ECOG scores, and have difficulty undergoing perfusion scintigraphy [9]. Utilizing CT scans to predict postoperative pulmonary function for these patients makes it easier to plan surgery and predict prognosis. CT scans showed higher accuracy in patients with high ECOG scores, thus providing a valuable method to evaluate patients who cannot cooperate with perfusion scintigraphy due to their poor condition. In addition, due to the recent coronavirus disease 2019 pandemic, the materials required for perfusion scintigraphy are not being imported, limiting the availability of these scans. In such situations, volumetry by CT can be a good substitute.

This study had several limitations. Although there were more than 50 patients who underwent pneumonectomy in our center, there was no protocol for perioperative lung function testing. Therefore, we had to exclude approximately 30 patients who did not have either preoperative or postoperative lung function testing. There were several lung function tests that did not include diffusing capacity of the lung for carbon monoxide (DLCO) and there were some with unreliable DLCO tests. Only 8 patients had both preoperative and postoperative DLCO values, which was too small a number for statistical analysis. The time interval between operation and postoperative lung function testing was inconsistent as well. As a result of this study, new protocols for lung resection operations were developed. Based on this investigation, a follow-up study is also planned for patients who underwent lobectomy.

No Korean studies were found on the prediction of postoperative lung function using CT volumetry. A few international studies were found using CT volumetry to predict postoperative lung function for lobectomy surgery. The design of this study was based on those studies, with results of similar significance. As the first domestic study using CT volumetry, this research will be expanded to include follow-up studies for lobectomy surgery. Additionally, a correlation with a high ECOG score was found, which is meaningful for patients with low performance. Further large-scale studies could help determine whether there is a correlation between ECOG and PPO-CT.

In conclusion, CT volumetry can predict pulmonary function precisely enough to plan surgery. In this study, an association with ECOG scores was also assessed based on the postoperative lung function predicted through CT volumetry in patients who underwent pneumonectomy. Based on advances in technology, it should also be possible to predict the postoperative lung function of patients who undergo lobectomy and segmentectomy and determine the relationship of ECOG scores with lung function.

\section{Conflict of interest}

No potential conflict of interest relevant to this article was reported.

\section{ORCID}

Hee Joon Kang: https://orcid.org/0000-0002-4161-6616

Seok Soo Lee: https://orcid.org/0000-0002-4402-0885

\section{References}

1. Luzzi L, Tenconi S, Voltolini L, et al. Long-term respiratory functional results after pneumonectomy. Eur J Cardiothorac Surg 2008; 34:164-8.

2. Smulders SA, Smeenk FW, Janssen-Heijnen ML, Postmus PE. Actual and predicted postoperative changes in lung function after pneumonectomy: a retrospective analysis. Chest 2004;125:1735-41.

3. Fernandez-Rodriguez L, Torres I, Romera D, et al. Prediction of postoperative lung function after major lung resection for lung cancer using volumetric computed tomography. J Thorac Cardiovasc Surg 2018;156:2297-308.

4. Wu MT, Chang JM, Chiang AA, et al. Use of quantitative CT to predict postoperative lung function in patients with lung cancer. Radiology 1994;191:257-62.

5. Bolliger CT, Guckel C, Engel H, et al. Prediction of functional reserves after lung resection: comparison between quantitative computed tomography, scintigraphy, and anatomy. Respiration 2002;69: 482-9.

6. Ueda K, Tanaka T, Li TS, Tanaka N, Hamano K. Quantitative computed tomography for the prediction of pulmonary function after lung cancer surgery: a simple method using simulation software. Eur J Cardiothorac Surg 2009;35:414-8.

7. Lamers RJ, Thelissen GR, Kessels AG, Wouters EF, van Engelshoven JM. Chronic obstructive pulmonary disease: evaluation with spirometrically controlled CT lung densitometry. Radiology 1994;193: 109-13.

8. Kinsella M, Muller NL, Abboud RT, Morrison NJ, DyBuncio A. 
Quantitation of emphysema by computed tomography using a "density mask" program and correlation with pulmonary function tests. Chest 1990;97:315-21.
9. Petersson J, Sanchez-Crespo A, Rohdin M, et al. Physiological evaluation of a new quantitative SPECT method measuring regional ventilation and perfusion. J Appl Physiol (1985) 2004;96:1127-36. 